\title{
Posterior Vaginal Wall Repair with Synthetic Absorbable Mesh: A New Technique for an Old Procedure
}

\author{
CAMIL CASTELO-BRANCO, M.D., JULIO F. GARRIDO, M.D., \\ CARLOS RIBAS, M.D., and XAVIER IGLESIAS, M.D.
}

\begin{abstract}
The objective of this study was to assess the applicability of posterior wall repair with a synthetic absorbable mesh. Between January and September 1996, five posterior repairs using absorbable synthetic meshes were performed. Five posterior wall repairs in patients matched for age, parity, and rectocele degree were performed according to usual procedures during the same period, and were used as controls. No febrile morbidity, cuff or posterior vaginal wall infections, thrombophlebitis, rectal injury, or hemorrhagic complications were observed in the 10 women who entered the study. In summary, posterior wall repair can be easily performed with an absorbable soft tissue patch, theoretically preserving sexual activity, and probably offers better functional results with longer experience, thus providing a safe and useful procedure in sexually active women. (J GYNECOL SURG 14:111, 1998)
\end{abstract}

\section{INTRODUCTION}

$\mathbf{P}$ OSTERIOR REPAIR IS PERFORMED in conjunction with perineorrhaphy to correct a rectocele and to reconstruct the perineal body. Basically, a rectocele is a hernia that develops when the perirectal adventitial tissue is insufficient to support the anterior rectal wall, and the rectum prolapses through the levator sling. The strength of the posterior vaginal mucosa is insufficient to prevent prolapse of the anterior rectal wall. The causes of this vaginal vault prolapse remain unclear. ${ }^{1}$

The purpose of classic posterior repair is to plicate the perirectal adventitial tissue and levator ani muscles over the anterior rectal wall and to provide a two-layer closure of this hernia. At this point, the redundant posterior vaginal mucosa is trimmed away. Following this technique, the vagina can be shorter and narrower, possibly making sexual relationships uncomfortable.

Soft tissue patches have been used widely in general surgery for wall defects correction. Nonabsorbable materials have often been preferred for such interventions because of their permanent strength. ${ }^{2,3}$ However, placing this kind of soft tissue patch close to the rectum may result in a conflictive rejection of the foreign-body graft, resulting in an immediate recurrence. ${ }^{4}$ Absorbable soft tissue mesh induces a perirectal fibrosis that may account for a stronger posterior wall, preserving the previous vaginal size. The aim of our study was to assess the practicality of posterior wall repair with a synthetic absorbable mesh.

Department of Gynecology and Obstetrics, Hospital Clínic i Provincial de Barcelona, Institut d'Investigació Biomèdica August Pi Suñer, Faculty of Medicine, Barcelona, Spain. 


\section{SUBJECTS AND METHODS}

\section{Patients}

Between January and September 1996, we performed 10 posterior vaginal vault repairs, five using polyglycolic acid mesh (synthetic absorbable mesh group) and five using reabsorbable sutures according to the classic procedure. The two groups were matched for age, parity, and rectocele degree. Data regarding patient characteristics in the two groups are given in Table 1.

Before inclusion in the study, the patients underwent medical history and physical examination. Only patients younger than 65 with moderate to severe rectocele who were sexually active were eligible. All selected patients gave their informed consent to this trial. After their inclusion and before the surgery, all of them underwent urethro-cysto-vagino-rectography (UCVRG) with barium contrast medium. One month after surgery, patients were examined for an initial clinical evaluation. Six and twelve months thereafter, the women were again evaluated clinically and radiologically with UCVRG.

\section{Technique}

After general or peridural anesthesia, the patient was placed in the dorsal lithotomy position with the buttocks well off the end of the table, and the vulva and vagina were fully prepared with an antiseptic solution. Following bladder catheterization, a bimanual examination was performed to differentiate between an enterocele and a rectocele; if there was doubt, a retractor was placed into the vagina to reveal the posterior wall, and a cleft could be observed between rectocele and enterocele. Evaluation of the perineal body was made to determine the extent of reconstruction needed.

The upper extent of the rectocele was identified, and two clamps were placed at the margins of the original hymen. An additional clamp was placed in the midline at the top of the rectocele. A transverse incision was made at the vulvar fourchette, and a Kelly clamp was inserted under the posterior vaginal wall,

Table 1. Patient Characteristics in the Two Groups

\begin{tabular}{lcc}
\hline Characteristic & Mesh & Reabsorbable suture \\
\hline Age (yr) & $57 \pm 7.1$ & $55.6 \pm 8.2$ \\
range & $46-64$ & $43-65$ \\
Para & & \\
1 & 1 & 0 \\
2 & 2 & 2 \\
$\geq 3$ & 2 & 3 \\
Menopausal status & 4 & 3 \\
Previous surgery & 3 & 2 \\
Abdominal hysterectomy & 2 & 0 \\
Vaginal hysterectomy & $1 \mathrm{~b}$ & $2^{\mathrm{c}}$ \\
Surgery & & \\
Posterior wall repair & 5 & 5 \\
Anterior wall repair & 2 & 4 \\
Abdominal wall hernia & 2 & 0 \\
Urine incontinence surgery & 1 & 1 \\
Vaginal hysterectomy & 1 & 3 \\
Operating time (min) & $107.6 \pm 24.6$ & $93 \pm 30.9$ \\
range & $45-135$ & $60-125$ \\
Hemoglobin before surgery & $13.5 \pm 0.6$ & $12.8 \pm 0.4$ \\
Hemoglobin after surgery & $11.6 \pm 1.2$ & $11.6 \pm 0.8$ \\
\hline
\end{tabular}

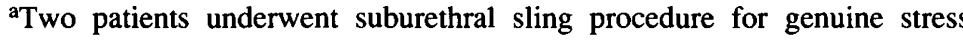
incontinence and low urethral closure pressure.

bThis patient underwent hysterectomy plus anterior and posterior wall repair.

cone patient underwent hysterectomy plus anterior and posterior wall repair and the other hysterectomy plus anterior wall repair. 
and the mucosa was dissected off the perirectal adventitial tissue. An additional incision was made in the perineal body, and a triangular piece of skin was removed. This exposed the insertion of the bulbocavernosus. Subsequently, a vertical incision in the posterior vaginal mucosa was made, and the edges were held with Kocher clamps. The perirectal adventitial tissue was dissected off the mucosa. The apex of the rectocele was held with an Allis clamp. At this point, a finger was placed over the rectocele, pushing it into the rectum, thus revealing the margins of the levator ani muscles. The next steps in the conventional surgery included the use of No. 1 absorbable interrupted sutures - we used polyglycolic acid-passed through the margins of the levator ani from the apex down to the posterior fourchette. Frequently this required 5 to 6 sutures to completely approximate the levator ani. Finally, the excessive posterior vaginal mucosa was trimmed away and a No. 0 synthetic absorbable continuous suture was placed at the apex of the vaginal mucosa and tied down. The closure of the posterior vaginal wall was completed to the posterior fourchette, and the former hymeneal ring was reconstructed. The vagina could, with this technique, become shorter and narrower.

Instead of these last steps, the use of synthetic absorbable mesh allowed correction of the rectocele and reconstruction of the vagina with less loss of vaginal mucosa. When the levator ani muscles were identified, and the rectocele was quite evident, the mesh was suited to the size of the rectocele (Fig. 1) and placed over and fixed with a No. 1 absorbable interrupted suture to the margins of both levator ani muscles from the apex down to the fourchette (Fig. 2). The closure of the posterior wall was completed without the removal of any vaginal mucosa, and vaginal capability was preserved.

\section{RESULTS}

Between January and September 1996, 10 patients with rectocele, aged 43 to 65 (mean 55.9 years), within 1 to 3 parity, and weighing $61-78 \mathrm{~kg}$, entered this study voluntarily. Five of these women underwent pos-

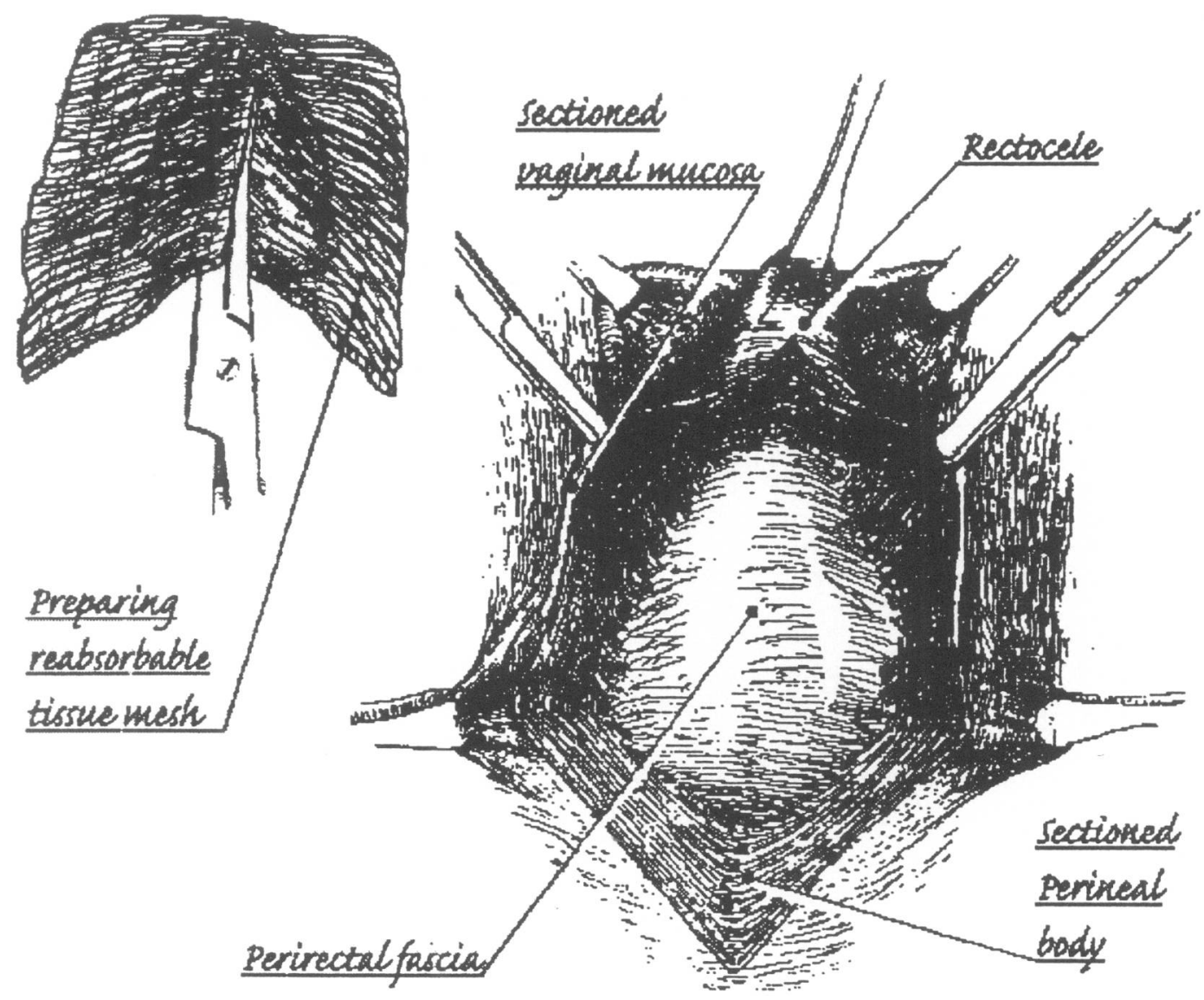

FIG. 1. The soft tissue patch is suited to the size of the rectocele. 


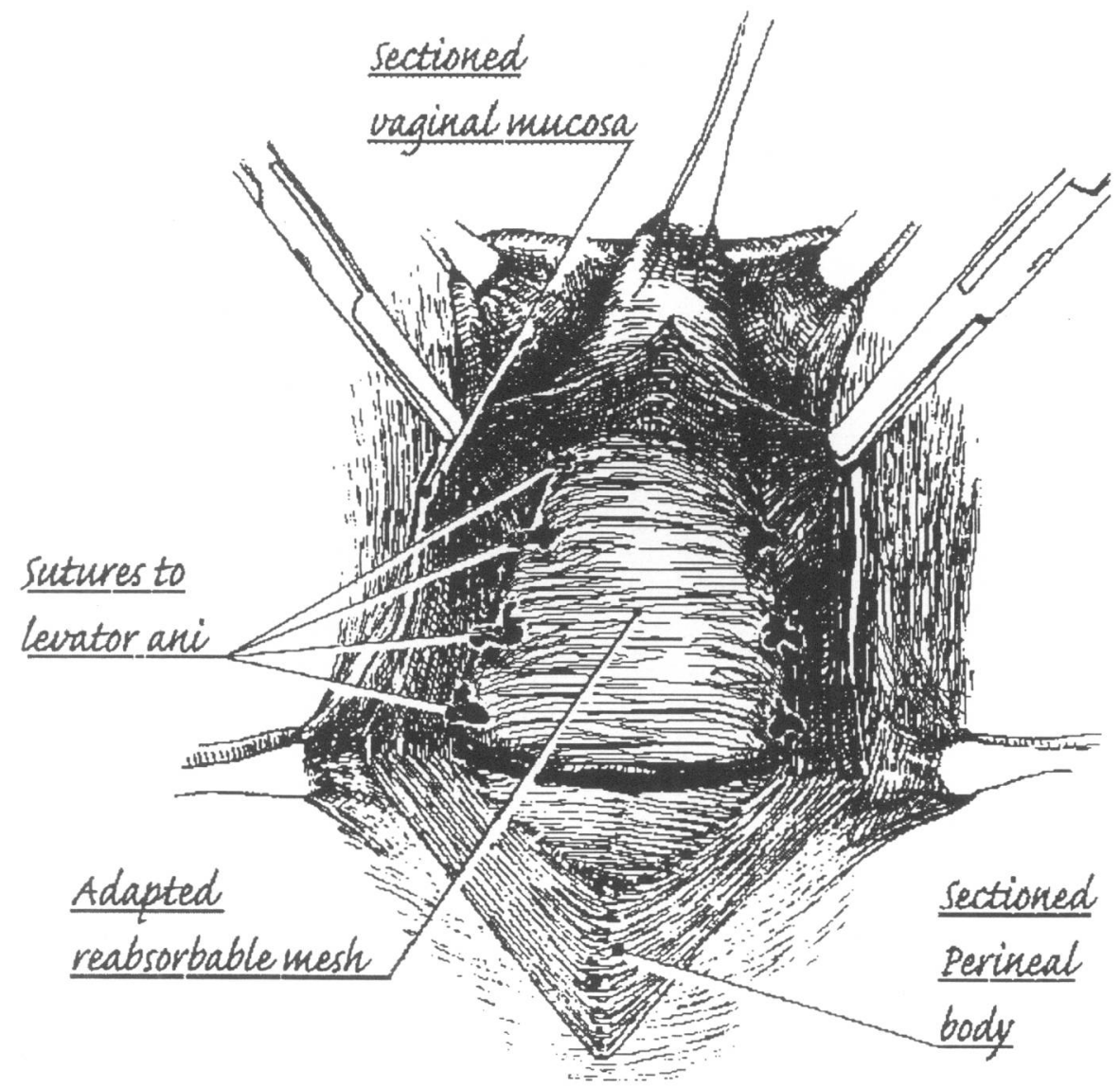

FIG. 2. The mesh is placed over the rectocele and fixed with a No. 1 absorbable interrupted suture to the margins of both levator ani muscles from the apex down to the fourchette.

terior repair with an absorbable synthetic soft tissue patch (group $M$ ), and the other five repairs were performed according to the usual procedures (group R). Three patients in group $\mathrm{M}$ and two in group $\mathrm{R}$ had undergone previous surgery (hysterectomy) before entering the study. Since most of the patients required more than one procedure during the surgery (Table 1), the range of operating time was wide (45-135 min). The clinical data, information from previous and present surgical procedures, operating times, and laboratory results are summarized in Table 1.

All the subjects were followed up 1 month after surgery, and patients were examined for an initial clinical evaluation. No complications occurred during surgery. No febrile morbidity was observed either in the soft tissue patch group or in the conventional surgery with reabsorbable sutures group. Only 3 cases of posterior wall granulomata were recorded ( 2 for the soft tissue patch group and 1 for the reabsorbable sutures group), and all of them were removed by torsion. In addition, one case of mild vulvar hematoma was observed in the conventional surgery group. No cuff or posterior vaginal vault infections, thrombophlebitis, rectal injury, or hemorrhage complications occurred.

Six and 12 months thereafter, the women again underwent clinical and radiologic evaluation. While preoperative UCVRG showed verticalization of the vagina according to the longitudinal axis of the patient induced by the rectocele (Fig. 3); 1 year after soft patch application, UCVRG showed that the rectum remained in a normal position and the vagina had conserved all its capability and size and recovered its normal course (Fig. 4). In addition, all the patients in group $M$ reported satisfactory sexual intercourse 1 year after surgery, whereas in two patients in group R, coitus was unpleasant because of the shortening of the vagina. 


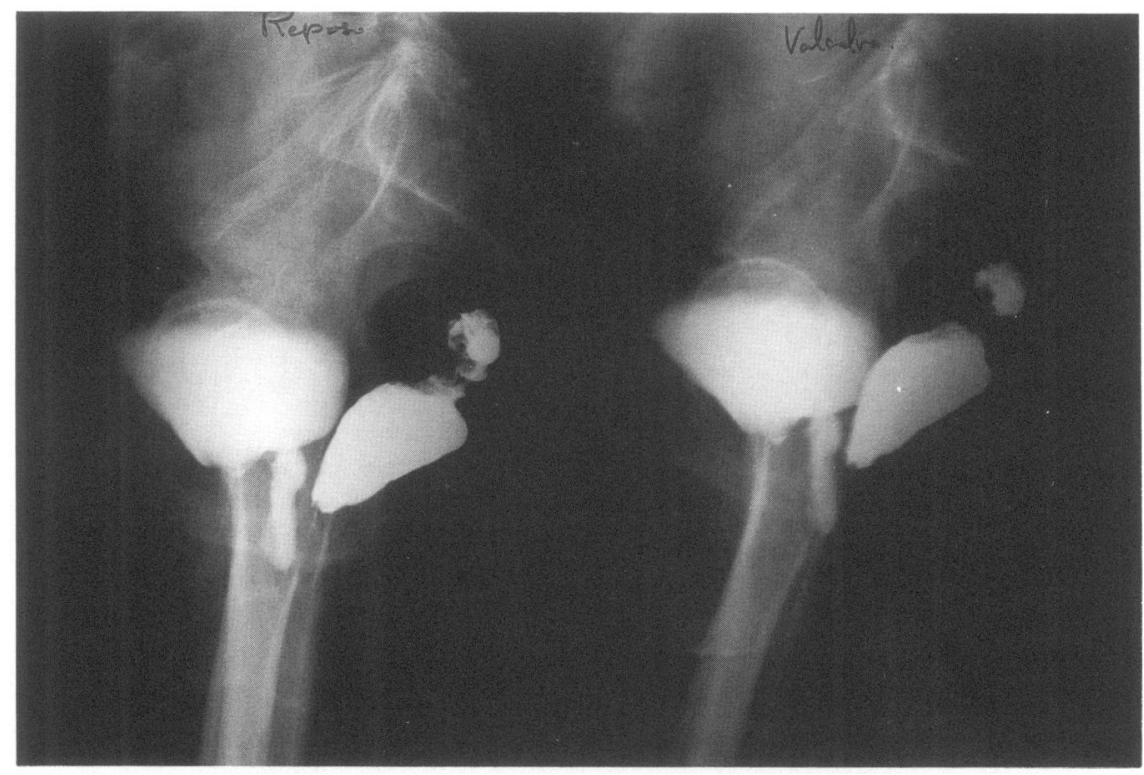

FIG. 3. Urethro-cysto-vagino-rectography in a patient with a severe rectocele before surgery. The vaginal course follows the longitudinal axis. During the Valsalva maneuver, the rectocele is more evident and is in contact with the anterior vaginal wall.

\section{DISCUSSION}

The management of vaginal vault prolapse has represented a therapeutic demand for which a multiplicity of surgical procedures have been developed over time. Nowadays, severe partial or complete prolapse of the vagina, the uterus, or both is a common challenge for the gynecologic surgeon. As a result

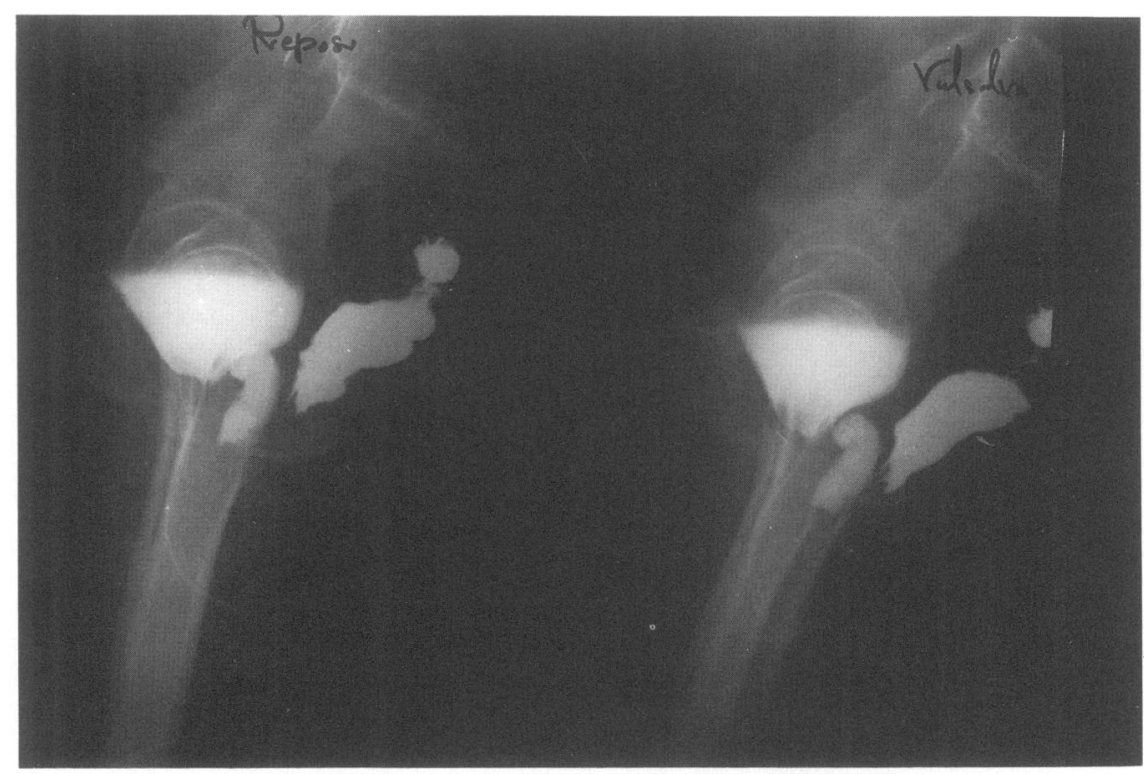

FIG. 4. Urethro-cysto-vagino-rectography in the same patient 1 year after surgery. The goal of reconstructive surgery has been achieved, and the vaginal course is now located in a better anatomic situation according to a cranioposterior axis. During the Valsalva maneuver, the posterior vaginal wall does not come into contact with the anterior vaginal wall. 
of changing viewpoints regarding sexuality and the psychologic and emotional value of the sexual organs, gynecologic surgeons must consider the wishes and opinions of the patient in preserving the function of the vagina.

In severe cases, posterior wall prolapse can be of such magnitude that coitus is impaired or impossible, and defecation becomes incomplete and difficult. To correct such a severe rectocele, but still allow satisfactory coitus, is a major endeavor. The successive introduction of new surgical approaches suggests continued disappointment with the results achieved, and additionally, repeated surgery may shorten or scar the vagina.

Another transient inconvenience of conventional surgery is that the levator ani muscles are included in the repair, and when this is done postoperatively, spasms can be present.

To supply a more stable and repair and decrease the risk of recurrence, several authors have suggested the use of synthetic nonreabsorbable material. ${ }^{2,3,5-10}$ However, the use of such materials has serious disadvantages. First, postoperative infections are impossible to cure without removal of the mesh; second, there is a risk of persistent vaginal fistula caused by the graft perforating the posterior vaginal wall; ${ }^{1}$ and finally, annoying delayed granulation reactions may appear up to 1 year later. ${ }^{4}$ Julian in a recent report ${ }^{9}$ assessed the efficacy and complications of Marlex mesh, a nonreabsorbable material, in repairing severe recurrent anterior vaginal wall prolapse, and although four patients in the control group and none in the treatment group experienced recurrent anterior vaginal wall prolapse, thus demonstrating the usefulness of such a procedure, three patients in the mesh group had mesh-related complications. Watson and colleagues ${ }^{10}$ demonstrated the use of Marlex mesh for transperineal repair of symptomatic rectocele. Eight of the nine women treated achieved successful evacuation after surgery without the need for vaginal digitation. Rectocele size and depth, and the percent of barium trapped in the rectocele on proctography, were all improved; however, anorectal physiology measurements were unchanged by surgery.

Iglesia et al., in an extensive review of the English literature from 1950 to the present, compared the properties of the most commonly used synthetic meshes and described their use in gynecologic procedures. ${ }^{11}$ The conclusions of their review were that long-term success with synthetic mesh ranges from $61 \%$ to $100 \%$; however, mesh-related complications rates are quite frequent, with up to a $35 \%$ removal rate, a $10 \%$ sinus tract formation rate, and a $9 \%$ erosion rate. For these reasons, Iglesia et al. reached the conclusion that the ideal synthetic mesh material for pelvic surgery, one that induces minimal foreign body reaction with a minimal risk of infection, rejection, and erosion, has yet to be developed.

On the other hand, the use of a polyglycolic acid mesh, a reabsorbable material, can solve these problems. Antibiotics can reach the mesh and prevent or cure the infection. Since the mesh is very soft and reabsorbable, vaginal fistula is not expected. Finally, although granulomatas may appear, they are usually small, asymptomatic, and easy to treat.

The polyglycolic acid mesh has become our material of choice for posterior vaginal wall restorative surgery because of several reasons, including those mentioned here. First, this material permits infiltration of fibroblast and a posterior fibrosis ${ }^{12}$ so that the grafted material becomes assimilated to the host anatomy. ${ }^{13}$ Theoretically, this can further reinforce the posterior wall. In addition, in a well-developed report, it has been suggested that the structure of the mesh is a major determinant of its degree of integration and the resistance to traction of the repaired zone, ${ }^{14}$ thus affecting the recurrence of prolapse. Second, polyglycolic acid has a high degree of acceptability by the human body. In our series, no case of rejection was recorded. Third, this material is very easy to manipulate and to cut according to requirements because of its flexibility and softness.

Finally, we recognize that our series is quite small and perhaps not large enough to enable the potential benefits and adverse effects of the mesh to be determined. However, our initial results encourage us to continue with this procedure. Of course, further studies are warranted.

\section{CONCLUSION}

Posterior wall repair can be performed with absorbable soft tissue patch easily, preserving the sexual activity, and probably offering better functional results, thus providing a useful procedure for sexually active women. 


\section{REFERENCES}

1. van Lindert ACM, Groenendijk AC, Scholten PC, Heintz APM. Surgical support and suspension of genital prolapse, including preservation of the uterus, using the gore-tex soft tissue patch: A preliminary report. Eur J Obstet Gynecol 1993;50:133.

2. Bremer EH. Prolapse of the vagina following total hysterectomy. Arch Surg 1966;92:20.

3. Durfee RB. Suspension operations for treatment of pelvic organ prolapse. Clin Obstet Gynecol 1966;9:1047.

4. Benson Harer W. Round ligament synthetic graft colpopexy. Obstet Gynecol 1994;83:1064.

5. Lane FE. Repair of posthysterectomy vaginal-vault prolapse. Obstet Gynecol 1962;20:72.

6. Symmonds RE, Sheldon RS. Vaginal prolapse after hysterectomy. Obstet Gynecol 1965;25:67.

7. Birnbaum SJ. Rational therapy for the prolapsed vagina. Am J Obstet Gynecol 1973;115:411.

8. Rust JA, Bottle JM, Howlett RJ. Prolapse of the vaginal vault: Improved techniques for the management of the abdominal approach or vaginal approach. Am J Obstet Gynecol 1976;125:768.

9. Julian TM. The efficacy of Marlex mesh in the repair of severe, recurrent vaginal prolapse of the anterior midvaginal wall. Am J Obstet Gynecol 1996;175:1472.

10. Watson SJ, Loder PB, Halligan S, Bartram CI, Kamm MA, Phillips RK. Transperineal repair of symptomatic rectocele with Marlex mesh: A clinical, physiological and radiologic assessment of treatment. J Am Coll Surg 1996;183:257.

11. Iglesia CB, Fenner DE, Brubaker L. Int Urogynecol J Pelvic Floor Dysfunct 1997;8:105.

12. Baykal A, Onat D, Rasa K, Renda N, Sayek I. Effects of polyglycolic acid and polypropylene meshes on postoperative adhesion formation in mice. World J Surg 1997;21:579.

13. Kyzer S, Kadouri A, Levi A, et al. Repair of fascia with polyglycolic acid mesh cultured with fibroblasts: Experimental study. Eur Surg Res 1997;29:84.

14. Bellón JM, Contreras LA, Buján J, Carrera-San Martín A. The use of biomaterials in the repair of abdominal wall defects: a comparative study between polypropylene meshes (Marlex) and a new polytetrafluoroethylene prosthesis (dual mesh). J Biomater Appl 1997;12:121.

Address reprint requests to: Camil Castelo-Branco

Department of Gynecology and Obstetrics Hospital Clínic i Provincial de Barcelona Villarroel 170 08036 Barcelona Spain 
This article has been cited by:

1. X Jia, C Glazener, G Mowatt, G MacLennan, C Bain, C Fraser, J Burr. 2008. Efficacy and safety of using mesh or grafts in surgery for anterior and/or posterior vaginal wall prolapse: systematic review and meta-analysis. BJOG: An International Journal of Obstetrics \& Gynaecology 115:11, 1350-1361. [CrossRef] 